\title{
CdSe-ZnO Core-Shell Quantum Dots for Protein Detection: A Potential Sensing Platform
}

\author{
Erin A. Jenrette ${ }^{1}$, Monique J. Farrell ${ }^{1}$, Jasmin A. Flowers ${ }^{1}$ and Aswini K. Pradhan ${ }^{1,2, *(D)}$ \\ 1 Center for Materials Research, Norfolk State University, 700 Park Ave., Norfolk, VA 23504, USA; \\ ejenrette@gmail.com (E.A.J.); monique.j.farrell@gmail.com (M.J.F.); sa.flowers@gmail.com (J.A.F.) \\ 2 Advance Material Solution, 2608 Horse Pasture Road, Suite 101, Virginia Beach, VA 23453, USA \\ * Correspondence: aswinigita2014@gmail.com
}

Citation: Jenrette, E.A.; Farrell, M.J.;

Flowers, J.A.; Pradhan, A.K.

CdSe-ZnO Core-Shell Quantum Dots for Protein Detection: A Potential Sensing Platform. Nanomanufacturing 2021, 1, 3-13. https://doi.org/ 10.3390/nanomanufacturing1010002

Academic Editor: Frank Shi

Received: 10 March 2021

Accepted: 6 April 2021

Published: 9 April 2021

Publisher's Note: MDPI stays neutral with regard to jurisdictional claims in published maps and institutional affiliations.

Copyright: (c) 2021 by the authors. Licensee MDPI, Basel, Switzerland. This article is an open access article distributed under the terms and conditions of the Creative Commons Attribution (CC BY) license (https:/ / creativecommons.org/licenses/by/ $4.0 /)$.

\begin{abstract}
A highly sensitive biosensing platform comprised of CdSe-ZnO core-shell nanostructures for targeted applications in protein detection is demonstrated. This innovative technique uses a microwave-assisted thermal decomposition method to produce a rapid, less hazardous, and userfriendly procedure to synthesize a semiconductor core surrounded by nanometer-thick metal oxide shells. The benefit of using a metal oxide shell includes mitigating the toxicity of the CdSe core, thus increasing its biocompatibility and minimizing its photochemical corrosion and oxidation. We present a simple one-pot microwave-assisted protocol for the formation of $\mathrm{CdSe}-\mathrm{ZnO}$ core-shell quantum dots (QDs). These QDs optimize the recognition limit of bovine serum albumin (BSA) protein through a spectral signal at a considerably low concentration $\left(2.5 \times 10^{-6} \mathrm{M}\right)$, thus demonstrating its potential to become a highly effective surface-plasmon-enhanced Raman spectroscopy (SERS)-like sensing platform. We report a QD material that can mimic a strong SERS-like behavior due to charge transfer affecting the local electric field.
\end{abstract}

Keywords: biosensing; quantum dots; surface plasmons; protein detection

\section{Introduction}

Due to the limited number of non-invasive detection methods currently available, it is essential to develop more reliable, non-invasive diagnostic systems with applications in pre-screening biomolecular detection. Currently, invasive procedures used to detect analytes can cause pain, discomfort, stress, and false positives. In addition to the adverse effects on health, the procedures are also time-intensive and cost-ineffective. Thus, there is a critical need for non-invasive diagnostics that are both accurate and quick. The development of a non-invasive method could potentially replace expensive conventional methods such as blood testing and biopsies. The core-shell structure described in this work offers a quick, invasive detection method, similar to nanotechnology-based devices such as a lab-on-a-chip [1]. This work focuses on the study of semiconductor nanocrystals/nanoparticles, which are known as quantum dots (QDs). Nanoparticles, specifically QDs, have unique optical properties that provide a strong platform for biological imaging, visual aids, and biosensing [2]. QDs are semiconducting nanoparticles that have quantumconfined holes and electrons that produce properties such as broad absorption bands, narrow emission bands, size-tunable emission, and extreme brightness [3]. Unlike other fluorescent molecules or fluorophores, QDs have sharper photoluminescence (PL) peaks, brighter fluorescence, and are more resistant to chemical degradation [4].

These unique optical properties of QDs allow them to be used for LEDs, drug delivery, and electronic and optoelectronic devices [5,6]. Although QDs have bright fluorescence and band gap tunability, they are quite toxic and oxidize easily. These limitations are compounded by their high surface-to-volume ratio, which decreases their quantum yield due to increased surface defects [2,7]. The most common method to prevent surface defects 
and enhance photostability is the synthesis of a metal oxide or semiconductor shell to surround the core. CdSe and $\mathrm{ZnO}$ were chosen as the core and metal oxide shell, respectively, because CdSe has good stability and high luminescence, whereas $\mathrm{ZnO}$ is biocompatible with a similar lattice structure to CdSe $[5,8]$. An et al. have shown shells of $\mathrm{ZnO}$ that can be successfully deposited by using a time-consuming and hazardous methodology [9]. We have improved on this process by using an alternative, high temperature, and quicker deposition method using a one-pot synthesis in the microwave. Furthermore, the detection limit of analytes has dramatically increased as a result of this new microwave-assisted method by fabricating a CdSe-ZnO core-shell nanosystem. This was demonstrated by using the protein (bovine serum albumin) BSA, which is one of the most abundant proteins found in the circulatory system and heavily contributes to osmotic blood pressure [10].

Raman spectroscopy has been a standard tool for the detection of biomolecules, including clinical settings [8]. However, due to the interference of fluorescence and the low detection limit of biomolecules, difficulty exists in identifying the proper structure of the analytes in the Raman spectra [11]. Thus, there is much room for improvement increasing the Raman signal by enhancing the detection limit of biomolecules using nanoparticles. The field of nanoparticles is a heavily researched topic due to their potential of high selectivity and sensitivity detection that can be achieved by taking advantage of a phenomenon called surface plasmon resonance (SPR) and localized SPR [12]. SPR can be used to enhance the detection of protein because it increases the surface sensitivity and enhancement factor, since each protein has a distinctive surface energy. Hence, the surface-enhanced Raman spectra (SERs) would allow the identification of analytes in the fingerprint region more effectively even at low concentrations, which has already been shown to enhance weak Raman signals as well as decrease the background noise of fluorescence [13-18]. Generally, SERs are observed in the presence of noble metals $(\mathrm{Au}, \mathrm{Ag}$, and $\mathrm{Cu})$ or alkali metal $(\mathrm{Na}, \mathrm{Li}$, and $\mathrm{K}$ ) surfaces with the excitation wavelength near the visible region $[19,20]$. However, other semiconducting materials have been proven to show Raman enhancement such as InAs/GaAs quantum dots, CuTe nanocrystals, $\mathrm{CuO}$ nanospheres, and $\mathrm{TiO}_{2}$ nanostructures in which charge transfer at the semiconductor-analyte interface plays a major role in Raman scattering enhancement [20-26].

In this study, we discuss an improved microwave-assisted approach for synthesizing CdSe-ZnO core-shell QDs, which led to their increased sensitivity for biosensing that was demonstrated by using a specific interaction between a protein and its aptamer. Additionally, our results further indicated Raman enhancement in semiconducting materials by protein attachment to a metal oxide shell $(\mathrm{ZnO})$ with CdSe core showing strong SERs-like behavior through Forster energy transfer (FRET) to ZnO from CdSe QDs. This suggests the possibility of using this platform as a sensing device for both biomedical as well as other thin film-based sensor applications.

\section{Materials and Methods}

The CdSe QDs were prepared by using a one-pot procedure in the microwave. In this synthesis, the selenium precursor was prepared by adding $0.03 \mathrm{~g}$ of selenium powder to 5 $\mathrm{mL}$ of 1-octadecene (ODE) and $400 \mu \mathrm{L}$ of trioctylphosphine (TOP). In a microwave glass vial, $10 \mathrm{~mL}$ of ODE and $600 \mu \mathrm{L}$ of oleic acid (OA) were added, and the vial was sealed. Then, the sealed glass vial was transferred to the glove box where $1 \mathrm{~mL}$ of the selenium precursor and $0.013 \mathrm{~g}$ cadmium oxide $(\mathrm{CdO})$ were added. Then, the sealed glass vial was removed from the glove box and transferred into the microwave (Anton Paar monowave 300, Anton Paar, Graz, Austria) where the nanocrystals were grown at $225^{\circ} \mathrm{C}$ for $3 \mathrm{~min}$. During the $3 \mathrm{~min}$ synthesis, $2 \mathrm{~mL}$ aliquots were taken every $30 \mathrm{~s}$ until the reaction was complete. The two-minute CdSe aliquot was used for $\mathrm{ZnO}$ shelling due to its highest concentration determined from photoluminescence.

The CdSe-ZnO core-shell nanocrystals were produced by the modifying method and calculations used by Nguyen et al. via a one-pot synthesis protocol in the microwave [6]. The $0.1 \mathrm{M} \mathrm{ZnO}$ precursor was prepared by adding $246 \mathrm{mg}$ zinc acteylacetonate $\left((\mathrm{acac})_{2}\right)$, 
$1.07 \mathrm{~g}$ oleylamine (OLA), and $6.84 \mathrm{~g}$ ODE to a microwave vial and synthesized in the microwave at $80{ }^{\circ} \mathrm{C}$ for $20 \mathrm{~min}$. Then, the precursor solution was maintained at $60{ }^{\circ} \mathrm{C}$ until it was ready for use. With the concentration of CdSe being $55 \mu \mathrm{M}$ and having a diameter of $2.57 \mathrm{~nm}, 543.49 \mu \mathrm{L}$ of CdSe was required for $\mathrm{ZnO}$ shelling. The calculations of the monomer requirements were based on the concentration of CdSe, $\mathrm{ZnO}$ density, $\mathrm{ZnO}$ molecular weight, $\mathrm{ZnO}$ lattice constant $(0.325 \mathrm{~nm})$, and the concentration of the $\mathrm{ZnO}$ precursor [6]. Depending on the desired monolayers (MLs) of $\mathrm{ZnO}$, the nanocrystals were prepared in a microwave vial by adding the required amount of the $\mathrm{ZnO}$ precursor needed for shelling, $3375 \mu \mathrm{L}$ of ODE, an equimolar amount of OA $(0.1 \mathrm{M})$, and $543.49 \mu \mathrm{L}$ of CdSe. For example, to deposit three monolayers of $\mathrm{ZnO}, 1125 \mu \mathrm{L}$ of the $\mathrm{ZnO}$ precursor solution was needed [6]. The $\mathrm{ZnO}$ shell was grown at $260^{\circ} \mathrm{C}$ for $30 \mathrm{~min}$ before cooling to room temperature. The schematic of this process is shown in Figure 1. The final core-shell material was washed through centrifugation using acetone/methanol and redispersed in hexane for characterization. All the chemicals used here are at least $99.99 \%$ purity.

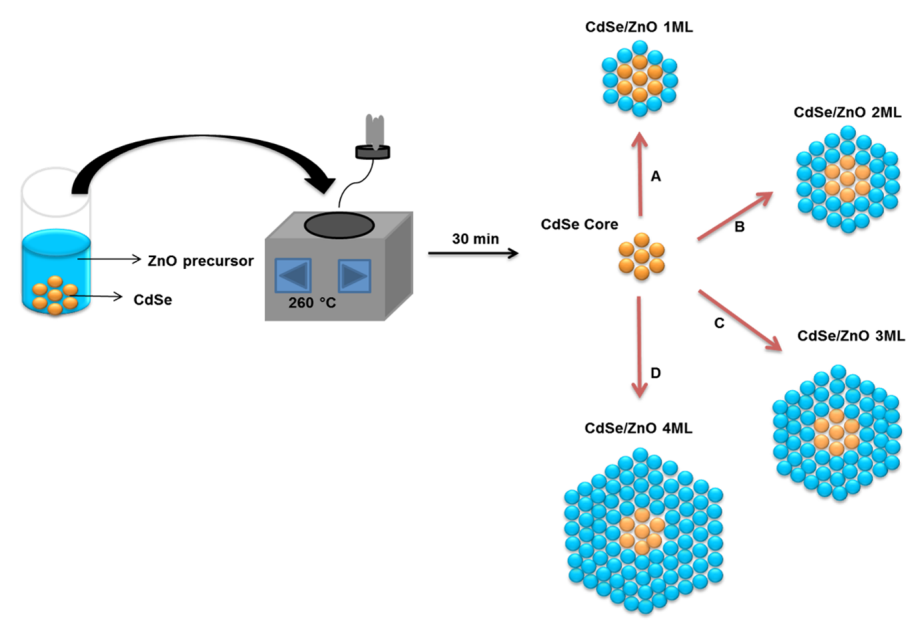

Figure 1. Schematic of the CdSe/ZnO nanostructure synthesis with one to four monolayers (MLs) $\mathrm{ZnO}$ shelling.

P-type (100) silicon substrates were ultrasonically cleaned in subsequent baths of trichloroethylene, acetone, and methanol $10 \mathrm{~min}$ each before silanization. The silanization process included drop casting a $1 \%$ solution of (3-aminopropyl) triethoxysilane (APTES) on the substrate, after which the solution was allowed to sit for $10 \mathrm{~min}$. After $10 \mathrm{~min}$, the substrate was rinsed with deionized (DI) water. Then, the core-shell nanoparticles were drop casted onto the substrate and allowed to sit for $10 \mathrm{~min}$ and rinsed again with DI water. Subsequently, a warm acetone solution $\left(50^{\circ} \mathrm{C}\right)$ was drop casted on the substrate and allowed to sit for approximately $30 \mathrm{~s}$ before rinsing the substrate with DI water followed by methanol. To complete the silanization process, the substrate was allowed naturally to dry.

The optical absorption of the core-shell material was recorded using a Perkin Elmer Lambda 950 UV-VIS-NIR spectrophotometer (Perkin Elmer, Waltham, MA, USA). The photoluminescence of the core-shell material was measured using a Hitachi F-700 fluorescence spectrophotometer (Hitachi, Tokyo, Japan). The morphology and microstructure of the as-synthesized materials were characterized by using a Hitachi 4700 transmission electron microscope (TEM) as well as a Rigaku Dmax 2200 X-ray diffractometer (Rigaku, Tokyo, Japan). The Raman spectra were acquired using a Horiba LabRam Confocal HR Evolution micro-Raman system (Horiba, Kyoto, Japan) with a 785 nm laser.

\section{Results and Discussions}

Figure 1 displays the schematic of the CdSe/ZnO core-shell nanostructure synthesis with 1 monolayer (ML) to 4 MLs of ZnO shelling. The UV-VIS absorbance spectra of the CdSe QDs are shown in Figure 2A. In order to evaluate the CdSe QDs microwave-assisted synthesis method, the absorbance spectra were monitored as a function of the reaction 
time. After thirty seconds of allowed reaction time, the first aliquot was removed, and the resulting absorbance spectrum was acquired, displaying a peak wavelength at $494 \mathrm{~nm}$ (Figures 2A and 3). As the reaction continued, subsequent aliquots were removed at thirty second intervals to produce a range of CdSe QDs with absorbance peak wavelengths ranging from 504 to $527 \mathrm{~nm}$, which are represented in Figure 3. In all cases, as the reaction time was increased, the peak absorbance wavelength was red shifted due to the increased size of the quantum dots [8]. We have demonstrated that this process uses an alternative, high-temperature, and quicker deposition method using a one-pot synthesis in the microwave with varying CdSe QDs size, and later, a $\mathrm{ZnO}$ precursor is used for shelling on the CdSe surface simultaneously.
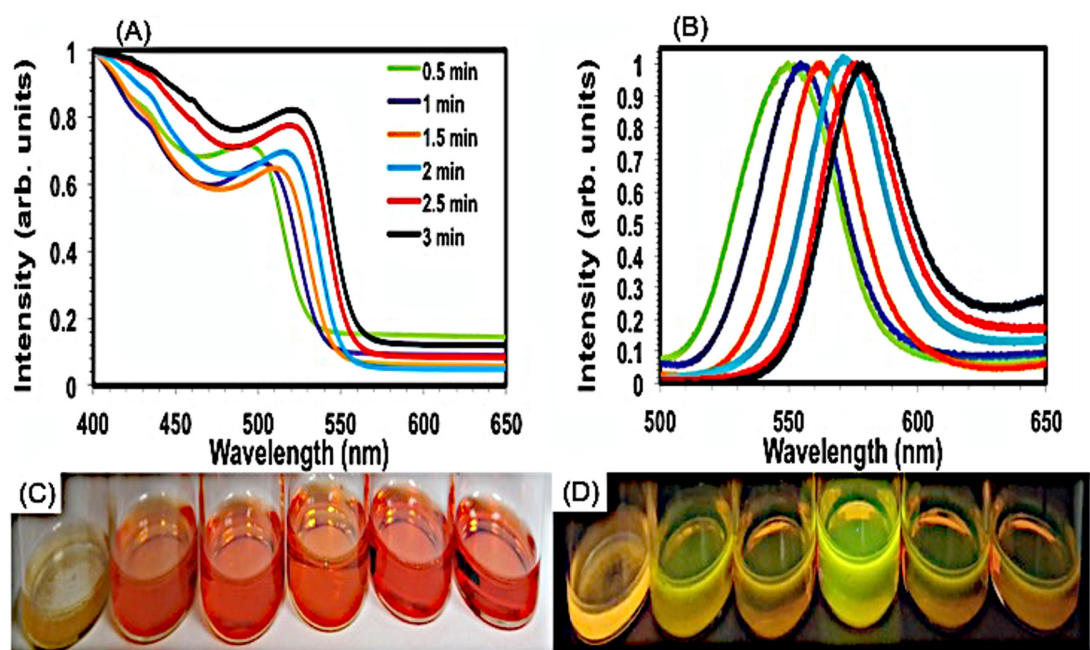

Figure 2. The characterization of UV-VIS absorption and photoluminescence (PL). The microwaveassisted thermal decomposition method allowed for the controlled growth of ZnO monolayers (MLs) around the CdSe core using a one-pot synthesis at $260^{\circ} \mathrm{C}$. (A) The normalized absorbance spectra and (B) the normalized photoluminescence spectra of the CdSe core aliquots. (C) Photographs of the respective aliquots under white light and (D) by UV light (365 nm).
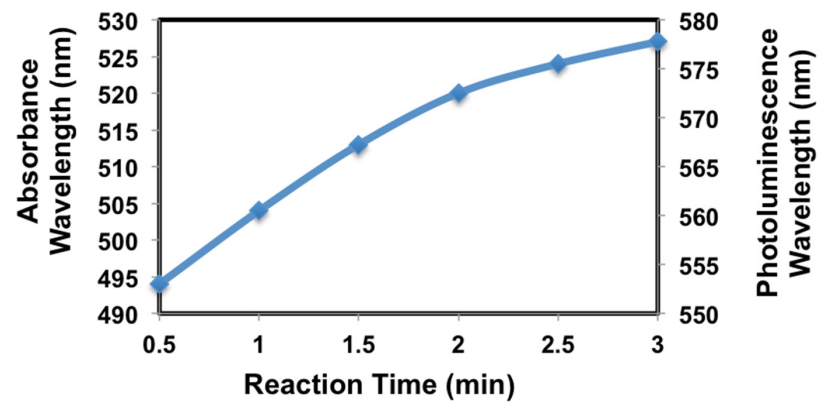

Figure 3. A curve representation of the UV-VIS and PL characterizations of the CdSe aliquots. The peak absorbance and photoluminescence wavelengths of the CdSe aliquots are depicted in Figure 2.

A very similar trend is also observed in the photoluminescence of the aliquots. Figure $2 B$ shows a monotonic red shift of the optical emission of the QDs as a function of their size due to extended reactions times, where photoluminescence peak wavelengths range from 552 to $578 \mathrm{~nm}$ and are represented in Figure 2B. The two-minute CdSe aliquot was used for $\mathrm{ZnO}$ shelling due to having the highest concentration determined by photoluminescence. The emission and absorption of color range with varying QDs size under white light and (E) UV light $(365 \mathrm{~nm})$ is shown in Figure 2C,D.

To further study the core-shell synthesis of CdSe- $\mathrm{ZnO}$, the absorbance and photoluminescence spectra were monitored as a function of the $\mathrm{ZnO}$ shell monolayer thickness (Figure 4A,B) using the two-minute CdSe core aliquot. There is a monotonic red-shifting 
that occurs in the absorbance and photoluminescence spectra of the core-shell system due to an increase in the overall nanoparticle size upon the addition of increasing concentrations of $\mathrm{ZnO}$. This red shift directly corresponds to the addition of $\mathrm{ZnO}$ MLs to the CdSe core.
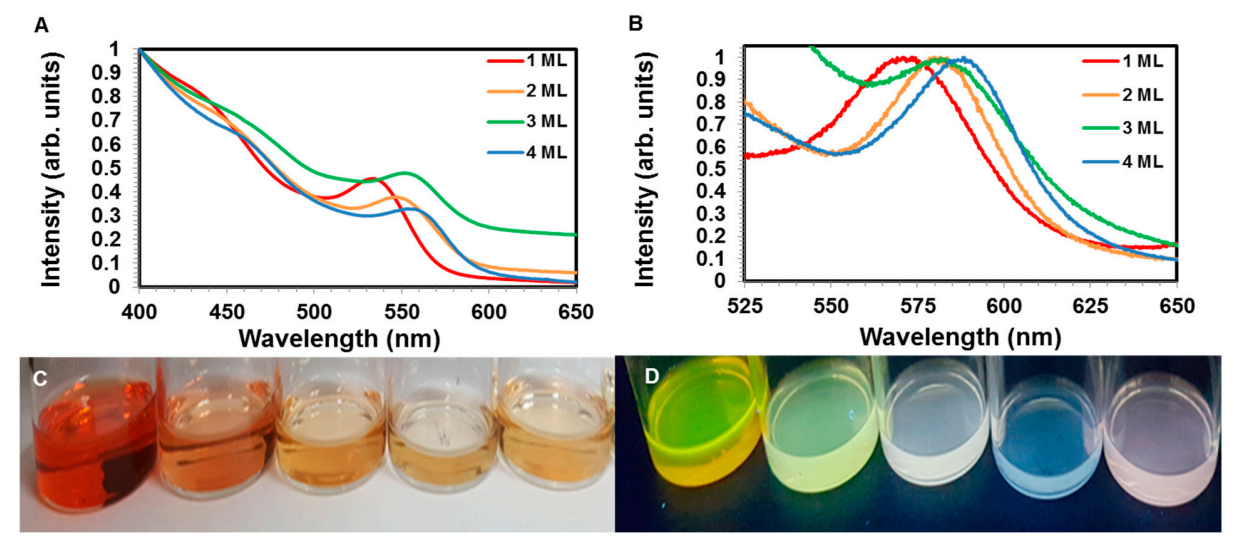

Figure 4. UV-VIS and PL characterizations of the CdSe-ZnO core-shell systems. (A) The normalized absorbance spectra and (B) the normalized photoluminescence spectra of the core-shell CdSe- $\mathrm{ZnO}$ with 1, 2, 3, and 4 MLs of ZnO. (C) Photographs of the CdSe core and the respective CdSe-ZnO solutions under white light and (D) UV light (365 nm) in consecutive order of ZnO MLs from left to right.

The maxima in absorbance peaks range from 537 to $561 \mathrm{~nm}$ and the photoluminescence peaks range from 572 to $589 \mathrm{~nm}$ as the $\mathrm{ZnO}$ monolayers are increased from one to four MLs, which are displayed in Table 1. Comparable to results reported elsewhere [5] that the full-width half-maxima (FWHM) of the narrow emission peaks increased from $28 \mathrm{~nm}$ for the CdSe two-minute aliquot core to $33 \mathrm{~nm}$ for the CdSe coated with four MLs of $\mathrm{ZnO}$ (shown the absorption spectra in supplementary Figure S1 and the corresponding photoluminescence spectra in Figure S2). Thus, the microwave-assisted method can alternatively be used to reproduce QDs-based systems with minimum trap states or defects. The increase of the FWHM of emission peaks can be attributed to the addition of the $\mathrm{ZnO}$ MLs, which naturally have broad trap emissions due to oxygen-related defects near the photoluminescence range of 450-550 $\mathrm{nm}$. The photoluminescence spectra of $\mathrm{ZnO}$ nanoparticles have characteristically displayed exciton emission approximately between 350 and $380 \mathrm{~nm}$ with some trap emission between 450 and $550 \mathrm{~nm}$ [5]. Consequently, the lattice mismatch between $\mathrm{CdSe}$ and $\mathrm{ZnO}$ can also attribute to the increase of full-width half-maxima (FWHM) due to the strain on the CdSe core upon the addition of $\mathrm{ZnO}$ shell thickness.

Table 1. Peak absorbance and photoluminescence wavelengths along with average particle sizes for the CdSe core ( $0 \mathrm{ML}$ of $\mathrm{ZnO}$ ) and CdSe-ZnO core-shell nanoparticles.

\begin{tabular}{cccc}
\hline $\begin{array}{c}\text { Number of ZnO } \\
\text { Monolayers }\end{array}$ & $\begin{array}{c}\text { Absorbance } \\
\text { Wavelength (nm) }\end{array}$ & $\begin{array}{c}\text { Photoluminescence } \\
\text { Wavelength (nm) }\end{array}$ & $\begin{array}{c}\text { Average Particle } \\
\text { Size (nm) }\end{array}$ \\
\hline 0 & 520 & 571 & 2.57 \\
\hline 1 & 537 & 572 & 2.80 \\
\hline 2 & 548 & 581 & 3.00 \\
\hline 3 & 554 & 583 & 3.12 \\
\hline 4 & 561 & 589 & 3.29 \\
\hline
\end{tabular}

The photoluminescence spectra of the core-shell CdSe-ZnO systems also revealed a strong enhancement and consistent red shifting of the CdSe QDs with increasing shell thickness. The core-shell CdSe-ZnO system with four MLs of ZnO displayed the highest intensity (see Figure S2). The strong enhancement and red shift indicate effective surface passivation of the core and an increased particle size upon the addition of ZnO MLS. The 
red shifting also confirms the lack of oxidation or reduction in the CdSe-ZnO system, which is normally exhibited in the CdSe-ZnS systems [27]. This clearly exhibits that the core CdSe QDs are intact. Therefore, further release of $\mathrm{Cd}$ and Se to the particle surface is not evident in the core-shell CdSe- $\mathrm{ZnO}$, mitigating the toxicity of this QD system and indicating better photostability than CdSe-ZnS. ZnS, a chalcogenide, is very susceptible to photoanodic oxidation, which can cause the shell layer to degrade. In addition, CdSe-ZnS has a lattice mismatch of $12 \%$, while $\mathrm{CdSe}-\mathrm{ZnO}$ has a drastically lower lattice mismatch of $7.1 \%[5,28]$. Due to having a higher lattice mismatch, the CdSe core is unable to withstand strain without forming defects in the CdSe-ZnS system when it reaches the maximum quantum yield at approximately 1.5 ML of shell thickness.

In Figure 2C,D, visual color changes support the addition of the $\mathrm{ZnO}$ layers and successful core-shell synthesis. The average particle sizes of the nanocrystals (see Table 1) were calculated by using the first absorption peak positions from the optical measurements in Figures 2A and 4A as well as from TEM images (see Figure 5). Yu et al. have shown the calculations of CdSe nanocrystal size by using empirical fitting functions [18]:

$$
\begin{gathered}
\text { CdSe: } \mathrm{D}=\left(1.6122 \times 10^{-9}\right) \lambda 4-\left(2.6575 \times 10^{-6}\right) \lambda 3+\left(1.6242 \times 10^{-3}\right) \lambda 2- \\
(0.4277) \lambda+(41.57) ;
\end{gathered}
$$

where $D(n m)$ is the size of a given nanocrystal sample and $\lambda(\mathrm{nm})$ is the wavelength of the first excitonic absorption peak [18].

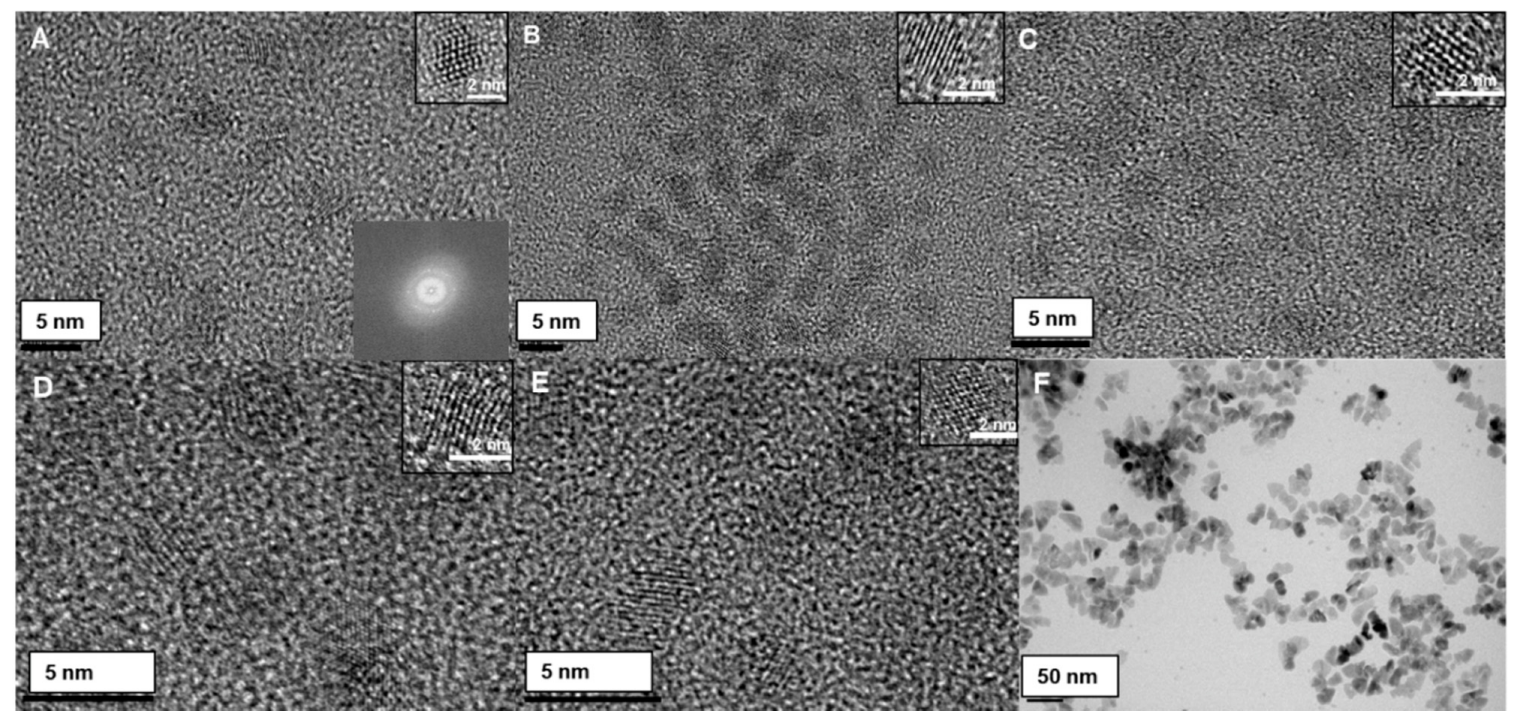

Figure 5. TEM images of the core CdSe, and the CdSe-ZnO core-shell systems. (A) CdSe core, and CdSe-ZnO core-shells with (B) one ML, (C) two MLs, (D) three MLs, (E) four MLs, and (F) five MLs of ZnO with corresponding high-resolution images (insets). A typical electron diffraction image for the QDs shows the polycrystalline hexagonal structures with preferred orientations (inset in A).

The successful core-shell synthesis is further supported by the real-space TEM images of the CdSe core and CdSe-ZnO core-shells shown in Figure 5. The QDs become larger in size with each additional $\mathrm{ZnO}$ layer, which corroborates to the red shift observed in Figures 2 and 4. Electron diffraction images (inset of Figure 5A) showed a polycrystalline hexagonal structure with preferred orientations, as expected for both $\mathrm{CdSe}$ and $\mathrm{ZnO}$, which were also observed from powder X-ray diffractometer (XRD) $\theta-2 \theta$ scans (not shown). Upon the addition of four MLs of $\mathrm{ZnO}$ (Figure 5E), the quantum dots have a clearly defined spherical shape, whereas CdSe coated with the five MLs of ZnO (Figure 5F) displays the expected tetrahedral morphology due to free wurtzite $\mathrm{ZnO}$ particles [5]. It is noted that the absolute intensity decreases slightly with the increase of $\mathrm{ZnO}$ monolayers. 
Raman spectroscopy was used to study the core-shell system of CdSe-ZnO QDs, since it is a standard tool to detect biomolecules. The Raman spectra of the CdSe-ZnO-4 MLs QDs on a silicon substrate are shown in Figure 6A. Although all CdSe-ZnO samples showed similar Raman peaks, the CdSe-ZnO-4 MLs system displayed the highest Raman intensity, and therefore, it was used for the biosensing platform in this study. The Raman modes of CdSe and $\mathrm{ZnO}$ are compared to previous literature in Table 2 [29-36] and are slightly shifted due to the shelling mechanism of the QDs [8]. For instance, the lower order longitudinal Raman modes of CdSe $\left(210 \mathrm{~cm}^{-1}\right.$ and $\left.228 \mathrm{~cm}^{-1}\right)$ are weakened and in some cases not observed due to the four MLs of $\mathrm{ZnO}$. Higher-order Raman peaks of CdSe are observed (425 and $607 \mathrm{~cm}^{-1}$ ), which represents the second-order phonon frequency and phonon mode 3LO1, respectively (Table 2). Correspondingly, the Raman features of $\mathrm{ZnO}$ are observed at 300,360,415,590, and $657 \mathrm{~cm}^{-1}$, which indicate the successful shelling of CdSe with $\mathrm{ZnO}$ (Table 2). The normal Raman spectrum of BSA with a concentration of $2.5 \times 10^{-4} \mathrm{M}$ was used as a control (baseline) to thoroughly characterize the core-shell QDs and its viability as a SERS sensor. Figure 6B shows that this BSA control has only four identifiable peaks $\left(940,980,1130\right.$, and $1244 \mathrm{~cm}^{-1}$ ) with very weak intensity (see Figure 7). Similarly, this trend was noticed in the lower concentrations of BSA, which included $2.5 \times 10^{-5} \mathrm{M}$ and $2.5 \times 10^{-6} \mathrm{M}$, thus indicating that the normal Raman has difficulties in identifying biomolecules. Using the core-shell QD system attached to the silicon substrate, we see an increase in the peak intensities observed in the control, as well as an increase in the total number of peaks specific to the biological molecule, BSA.
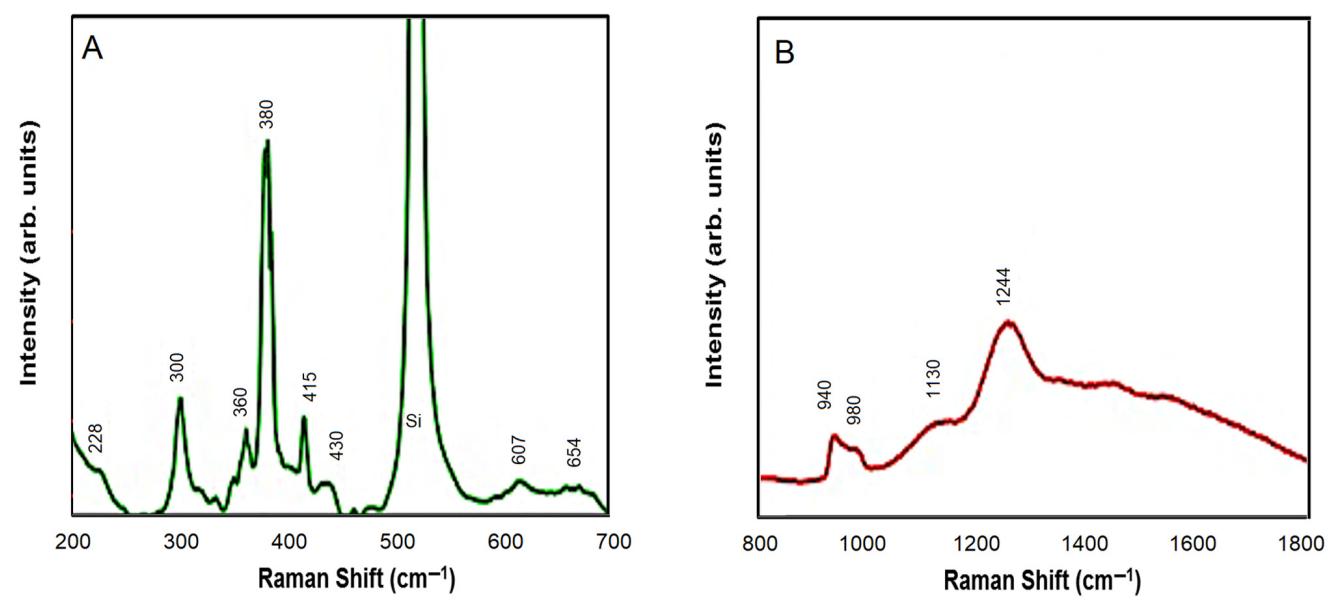

Figure 6. Raman spectra of CdSe-ZnO-4 MLs core-shell system and $2.5 \times 10^{-4} \mathrm{M}$ bovine serum albumin (BSA). (A) The CdSe-ZnO core-shell system with four MLs of $\mathrm{ZnO}$ on a silicon substrate (green) and (B) $2.5 \times 10^{-4} \mathrm{M}$ BSA on a silicon substrate (red).

Table 2. The Raman modes of CdSe and the corresponding CdSe modes found in the as-prepared microwave-synthesized $\mathrm{CdSe}-\mathrm{ZnO}$ core-shell system with four MLs of $\mathrm{ZnO}$, and the Raman modes of $\mathrm{ZnO}$ found in the as-prepared microwave CdSe-ZnO core-shell system with four MLs of $\mathrm{ZnO}$.

\begin{tabular}{|c|c|c|c|c|}
\hline $\begin{array}{c}\text { Number of } \\
\text { Raman Modes }\end{array}$ & $\begin{array}{c}\text { CdSe Modes }\left(\mathrm{cm}^{-1}\right) \\
{[29-36]}\end{array}$ & $\begin{array}{l}\text { Peaks Observed from } \\
\text { CdSe-ZnO }\left(\mathrm{cm}^{-1}\right)\end{array}$ & $\begin{array}{c}\text { ZnO Modes }\left(\mathrm{cm}^{-1}\right) \\
{[37,38]}\end{array}$ & $\begin{array}{l}\text { Peaks Observed from } \\
\text { CdSe-ZnO }\left(\mathrm{cm}^{-1}\right)\end{array}$ \\
\hline 1 & 210 & 228 & 284 & 300 \\
\hline 2 & 228 & 430 & 332 & 360 \\
\hline 3 & 425 & 607 & 378 & 380 \\
\hline 4 & 607 & - & 410 & 415 \\
\hline 5 & - & - & 438 & 430 \\
\hline 6 & - & - & 590 & - \\
\hline 7 & - & - & 618 & - \\
\hline 8 & - & - & 657 & 654 \\
\hline
\end{tabular}




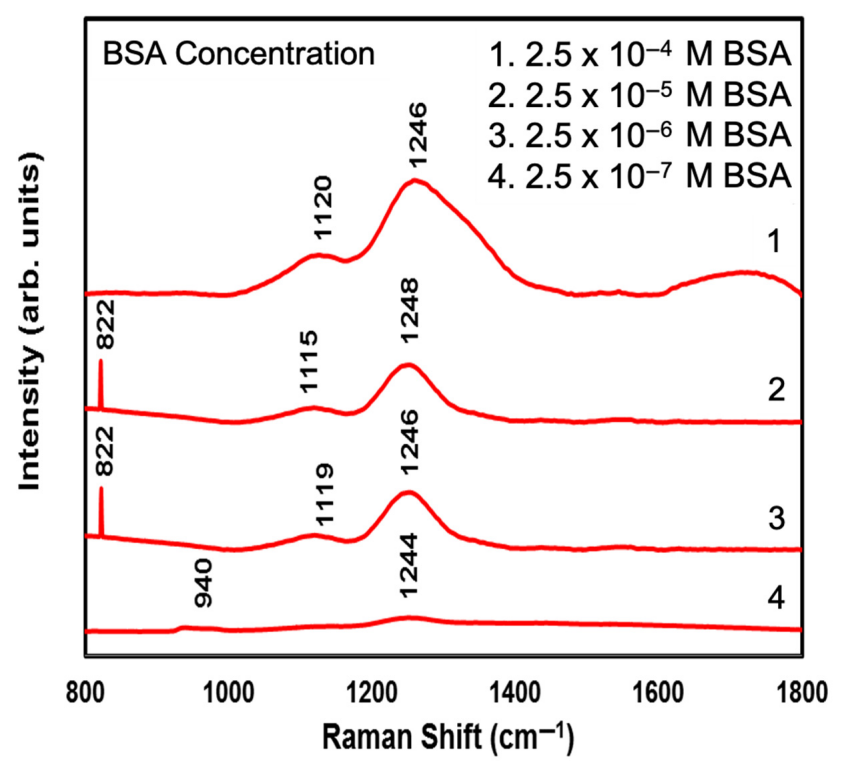

Figure 7. The Raman spectra of the CdSe/ZnO system with four MLs on a silicon substrate with different BSA concentrations, $2.5 \times 10^{-4} \mathrm{M}$ BSA (top) to $2.5 \times 10^{-7} \mathrm{M}$ BSA (bottom).

Different concentrations of solution were prepared at room temperature from BSA powders, and the solutions were stored in freeze at about $40 \mathrm{~F}$. As several factors affect protein assay accuracy and precision, we included replicates of each standard and test samples. The standards were run three times. The results were found reproducible in each run. The BSA protein was drop casted on the silicon substrates without the quantum dot systems and $\mathrm{CdSe} / \mathrm{ZnO}$ substrates with the incubation times ranging from $10 \mathrm{~min}$ to $24 \mathrm{~h}$. Following the incubation, the excess protein was spin coated off at various speeds (5000-6000 RPM) and times (10-30 s). Utilizing the same incubation times, the spin-coating step was also omitted for another batch of the same samples to determine if the Raman intensity would be affected scanning a wet substrate containing excess protein. It was concluded that the Raman intensities were independent of the protein incubation time and deposition method. This suggested that these devices could potentially be used for real-time analysis studies. However, the Raman studies showed higher intensities for the dry substrates with less fluorescence noise compared to the wet substrates.

The Raman spectrophotometer could only distinguish six peaks, whereas one of those peaks signified the presence of the silicon substrate. However, upon use of the core-shell system attached to the p-type bulk silicon, we see an increase within the intensities of the peaks observed and the number of peaks specific to the biological molecule present. In Figure 7, we present that as low as $2.5 \times 10^{-7} \mathrm{M}$ BSA (bottom) concentration is detectable using the current Raman experiment. Upon use of the core-shell CdSe/ZnO QD system attached to the silicon substrate, we see an increase in the peak intensities observed in the control as well as an increase in the total number of peaks specific to BSA protein. Compared to $2.5 \times 10^{-4} \mathrm{M}$ BSA on a CdSe/ZnO-4 MLs core-shell QD-coated silicon substrate vs. $2.5 \times 10^{-4} \mathrm{M}$ BSA on a bare silicon substrate, the Raman intensity varies from 20,000 (a.u.) to 1000 (a.u.) under the same conditions. It is very distinct that the Raman intensity significantly increases with the increase of BSA to $2.5 \times 10^{-4} \mathrm{M}$, as shown in Figure 7. The Raman intensity is enhanced by order of magnitude, which is discussed below under the Forster energy transfer (FRET) [3,8] mechanism.

In Figure 8, we observe that utilizing our core-shell QD system successfully detected approximately eight peaks specific to BSA as opposed to just four peaks without the employment of our QD system. The Raman band assignment peaks of BSA without and with the presence of the CdSe-ZnO-4 MLs core-shell system is shown in Table 3. The corresponding BSA peaks were strongly enhanced on our biosensing platform even at the considerable low concentration of $2.5 \times 10^{-6} \mathrm{M}$. This enhancement can be attributed 
to the SERs theory because it implies there is a proximity-induced excitation of localized surface plasmons [29-38]. Both Raman modes in CdSe and $\mathrm{ZnO}$ contributed to enhanced fingerprints, which produced hot spots suggesting SERs-like activity on our platform through FRET to ZnO from CdSe QDs [3,8]. The energy transfer explains FRET from QD-based donor-excited states to a proximal acceptor through nonradiative dipole-dipole coupling. However, the energy transfer efficiency between the donor-acceptor depends on the distance, relative orientation, and the overlap between the donor emission and acceptor absorption spectra. Our approach of biosensor platform is comparable to some other biosensors [39-43]. However, detailed work is necessary to evaluate the figure of merit and sensitivity of the system, although the current studies show the detection limit better than $10^{-6}$.

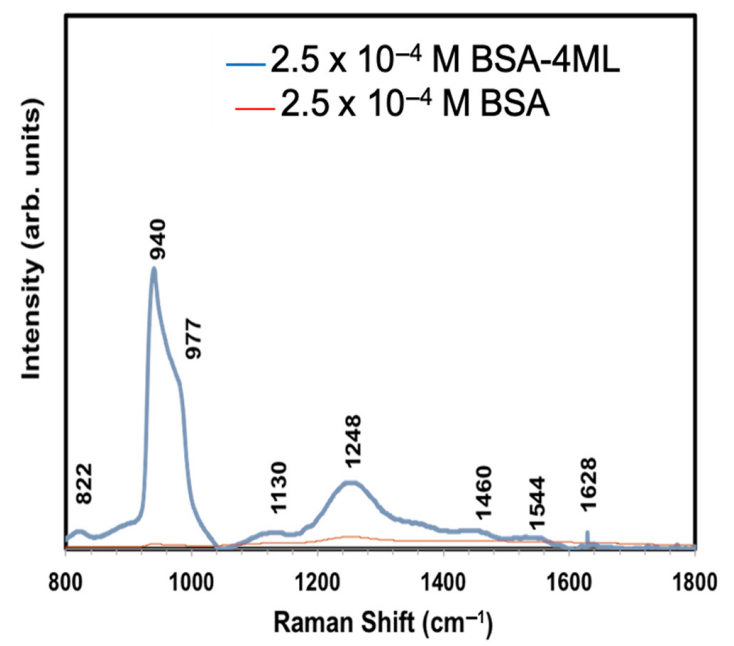

Figure 8. Raman spectra of $2.5 \times 10^{-4} \mathrm{M}$ BSA on a CdSe-ZnO-4 MLs core-shell QD-coated silicon substrate (blue) vs. $2.5 \times 10^{-4} \mathrm{M}$ BSA on a bare silicon substrate (red).

Table 3. Comparison of the Raman band assignment peaks of BSA without and with the presence of the CdSe-ZnO-4 MLs core-shell system.

\begin{tabular}{|c|c|c|c|}
\hline $\begin{array}{c}\text { Number of } \\
\text { Raman Modes }\end{array}$ & $\begin{array}{c}2.5 \times 10^{-4} \text { M BSA on a Si } \\
\text { Substrate }\left(\mathrm{cm}^{-1}\right)\end{array}$ & $\begin{array}{l}2.5 \times 10^{-4} \mathrm{M} \text { BSA on a CdSe-ZnO-4 } \\
\text { ML Coated Si Substrate }\left(\mathrm{cm}^{-1}\right)\end{array}$ & $\begin{array}{c}\text { Band Assignment Peaks of } \\
\text { BSA }\left(\mathrm{cm}^{-1}\right)[39,40]\end{array}$ \\
\hline 1 & 940 & 822 & 822 \\
\hline 2 & 980 & 940 & 940 \\
\hline 3 & 1130 & 977 & 979 \\
\hline 4 & 1244 & 1130 & 1127 \\
\hline 5 & - & 1248 & 1254 \\
\hline 6 & - & - & 1359 \\
\hline 7 & - & 1460 & 1458 \\
\hline 8 & - & 1544 & 1530 \\
\hline 9 & - & 1628 & 1633 \\
\hline
\end{tabular}

\section{Conclusions}

We have demonstrated the detection of low concentrations of BSA using a CdSe$\mathrm{ZnO}$ biosensing platform that was formed by a novel microwave-assisted procedure. Our experimental results prove that this procedure produces highly crystalline core-shell QDs with varying $\mathrm{ZnO}$ monolayers. A monotonic red shifting was observed in the absorbance and photoluminescence spectra of the core-shell system due to an increase in the overall particle size upon the addition of increasing concentrations of $\mathrm{ZnO}$, which directly related to increasing MLs. The addition of the ZnO MLs could be visually seen under both white and UV light. The addition of four MLs exhibited a clearly defined, spherical shape that measured $3.29 \mathrm{~nm}$ in diameter. The uniform distribution of these QDs created hot spots in 
the spaces in between, making them ideal for SERs-like detection, which became evident in the Raman spectra. To test the SERs-like capabilities of this platform, we optimized the detection limit of BSA and detected concentrations as low as $2.5 \times 10^{-6} \mathrm{M}$ using Raman spectroscopy. The intensity of the spectral signal of BSA alone was drastically enhanced when it was on the core-shell QD platform due to efficient energy transfer from the core to the $\mathrm{ZnO}$ shell. This nanoparticle core-shell system has the potential to become a highly sensitive SERS-like substrate and has also demonstrated extended capabilities to be used for thin film applications.

Supplementary Materials: The following are available online at https:/ /www.mdpi.com/2673-687 X/1/1/2/s1, Figure S1: The absorption spectrum of CdSe two-minute aliquot QDs and the core-shell CdSe-ZnO with four MLs of $\mathrm{ZnO}$, Figure S2: The PL spectrum of CdSe two-minute aliquot QDs and the core-shell CdSe- $\mathrm{ZnO}$ with one and four MLs of $\mathrm{ZnO}$.

Author Contributions: Funding acquisition by A.K.P.; E.A.J. and A.K.P. conceived the idea. E.A.J., M.J.F., and J.A.F. conducted the experiments. E.A.J. and A.K.P. wrote the manuscript. All authors have read and agreed to the published version of the manuscript.

Funding: This work was supported by the National Science Foundation Centers of Research Excellence in Science and Technology (NSF-CREST) Grant Number HRD 1036494 and 1547771, and NASA Office of Education Minority University Research and Education Project (OE MUREP) Advanced STEM Training and Research (ASTAR) Fellowship Grant Number NNX15AU34H. All the funded projects were managed by the Project Director, AKP.

Acknowledgments: The authors would like to thank Wei Cao for help in TEM imaging.

Conflicts of Interest: The authors declare no conflict of interest.

\section{References}

1. Xiao, B.; Hu, R.; Lin, G.; Roy, I.; Yong, K.-T. Enhanced optical transmission and fano resonance through a nanostructured metal thin film. Sci. Rep. 2015, 5, 10393. [CrossRef]

2. Chen, M.L.; Hu, R.; Lin, G.; Roy, I.; Yong, K.-T. Quantum-dot-conjugated graphene as a probe for simultaneous cancer-targeted fluorescent imaging, tracking, and monitoring drug delivery. Bioconjugate Chem. 2013, 24, 387-397. [CrossRef]

3. Wang, Y.; Hu, R.; Lin, G.; Roy, I.; Yong, K.-T. Functionalized quantum dots for biosensing and bioimaging and concerns on toxicity. Acs Appl. Mater. Interfaces 2013, 5, 2786-2799. [CrossRef]

4. Dondapati, H.; Ha, D.; Jenrette, E.; Xiao, B.; Pradhan, A.K. High-performance chemical-bath deposited CdS thin-film transistors with $\mathrm{ZrO}_{2}$ gate dielectric. Appl. Phys. Lett. 2014, 105, 052105. [CrossRef]

5. Nguyen, T.L.; Margaret, M.; Paul, M. Synthesis of highly crystalline CdSe@ZnO nanocrystals via monolayer-by-monolayer epitaxial shell deposition. Chem. Mater. 2014, 26, 4274-4279. [CrossRef]

6. Dondapati, H.; Ha, D.; Pradhan, A.K. Enhanced photocurrent in solution processed electronically coupled CdSe nanocrystals thin films. Appl. Phys. Lett. 2013, 103, 121114. [CrossRef]

7. Alivisatos, A.P. Semiconductor clusters, nanocrystals, and quantum dots. Science 1996, 271, 933. [CrossRef]

8. Jenrette, E.; Pradhan, S.K.; Rutherford, G.; Flowers, J.; Ha, D.; Pradhan, A.K. Quantum-dot-conjugated graphene oxide as an optical tool for biosensor. Opt. Express 2015, 23, 25017-25027. [CrossRef]

9. An, L.M.; Chen, X.L.; Han, X.T.; Yi, J.; liu, C.X.; An, W.Y.; Qu, Y.Q.; Chi, J.G.; Wei, H.; Qi, K.Y. CdSe/ZnO core/shell semiconductor nanocrystals: Synthesis and characterization. Appl. Mech. Mater. 2012, 268, 207-210. [CrossRef]

10. Chakraborty, T.; Chakraborty, I.; Moulik, S.P.; Ghosh, S. Physicochemical and conformational studies on BSA-Surfactant interaction in aqueous medium. Langmuir 2009, 25, 3062-3074. [CrossRef] [PubMed]

11. Efremov, E.V.; Freek, A.; Cees, G. Achievements in resonance raman spectroscopy: Review of a technique with a distinct analytical chemistry potential. Anal. Chim. Acta 2008, 606, 119-134. [CrossRef]

12. Liu, Y.; Liu, Q.; Chen, S.; Cheng, F.; Wang, H.; Peng, W. Surface plasmon resonance biosensor based on smart phone platforms. Sci. Rep. 2015, 5, 12864. [CrossRef] [PubMed]

13. Wang, Y.; Luo, Z.; Liu, K.; Wang, J.; Duan, Y. In situ targeting TEM8 via immune response and polypeptide recognition by wavelength-modulated surface plasmon resonance biosensor. Sci. Rep. 2016, 6, 20006. [CrossRef]

14. Reyes-Goddard, J.M.; Hugh, B.; Nicholas, S. Photodiagnosis using raman and surface enhanced raman scattering of bodily fluids. Photodiagnosis Photodyn. Ther. 2005, 2, 223-233. [CrossRef]

15. Chiu, N.F.; Teng-Yi, H.; Hsin-Chih, L. Graphene oxide-based surface plasmon resonance biosensors. Adv. Graphene Sci. InTech. 2013, 280, 191-216.

16. Liu, Z.; Guo, Z.; Zhong, H.; Qin, X.; Wan, M.; Yang, B. Graphene oxide based enhanced raman scattering probes for cancer cell imaging. Phys. Chem. Chem. Phys. 2013, 15, 2961-2966. [CrossRef] 
17. He, S.; Liu, K.-K.; Su, S.; Yan, J.; Mao, X.; Wang, D.; He, Y.; Li, L.-J.; Song, S.; Fan, C. Graphene-based high-efficiency surfaceenhanced raman scattering-active platform for sensitive and multiplex DNA detection. Anal. Chem. 2012, 84, 4622-4627. [CrossRef] [PubMed]

18. Yu, W.W.; Qu, L.; Guo, W.; Peng, X. Experimental determination of the extinction coefficient of CdTe, CdSe and CdS Nanocrystals. Chem. Mater. 2004, 16, 560. [CrossRef]

19. Zeman, E.J.; Schatz, G.C. An accurate electromagnetic theory study of surface enhancement factors for silver, gold, copper, lithium, sodium, aluminum, gallium, indium, zinc, and cadmium. J. Phys. Chem. 1987, 91, 634-643. [CrossRef]

20. Cong, S.; Yuan, Y.; Chen, Z.; Hou, J.; Yang, M.; Su, Y.; Zhang, Y.; Li, L.; Li, Q.; Geng, F.; et al. Noble metal-comparable SERS enhancement from semiconducting metal oxides by making oxygen vacancies. Nat. Commun. 2015, 6, 7800-7801. [CrossRef]

21. Quagliano, L.G. Observation of molecules adsorbed on IIIV semiconductor quantum dots by surface enhanced Raman scattering. J. Am. Chem. Soc. 2004, 126, 7393-7398. [CrossRef] [PubMed]

22. Li, W.; Zamani, R.; Gil, P.R.; Pelaz, B.; Ibanez, M.; Cadavid, D.; Shavel, A.; Alvarez-Puebla, R.A.; Parak, W.J.; Arbiol, J. CuTe nanocrystals: Shape and size control, plasmonic properties, and use as SERS probes and photo thermal agents. J. Am. Chem. Soc. 2013, 135, 7098-7101. [CrossRef] [PubMed]

23. Jiang, L.; You, T.; Yin, P.; Shang, Y.; Zhang, D.; Guo, L.; Yang, S. Surface enhanced raman scattering spectra of adsorbates on CuO nanospheres: Charge transfer and electromagnetic enhancement. Nanoscale 2013, 5, 2784-2789. [CrossRef]

24. Musumeci, A.; Gosztola, D.; Schiller, T.; Dimitrijevic, N.M.; Mujica, V.; Martin, D.; Rajh, T. SERS of semiconducting nanoparticles (TiO hybrid composites). J. Am. Chem. Soc. 2009, 131, 6040-6041. [CrossRef] [PubMed]

25. Qi, D.; Lu, L.; Wang, L.; Zhang, J. Improved SERS sensitivity on plasmon free $\mathrm{TiO}_{2}$ photonic microarray by enhancing light matter coupling. J. Am. Chem. Soc. 2014, 136, 9886-9889. [CrossRef]

26. Li, L.; Hutter, T.; Finnemore, A.S.; Huang, F.M.; Baumberg, J.J.; Elliott, S.R.; Steiner, U.; Mahajan, S. Metal oxide nanoparticle mediated enhanced raman scattering and its use in direct monitoring of interfacial chemical reactions. Nano Lett. 2012, 12, 4242-4246. [CrossRef]

27. Roy, D.; Routh, T.; Asaithambi, A.V.; Mandal, S.; Mandal, P.K. Spectral and temporal optical behavior of blue-, green-, orange-, and red-emitting CdSe-based core/gradient alloy shell/shell quantum dots: Ensemble and single-particle investigation results. J. Phys. Chem. C 2016, 120, 3483-3491. [CrossRef]

28. Andrew, S.; Aaron, M.; Mohs, M.; Nie, S. Tuning the optical and electronic properties of colloidal nanocrystals by lattice strain. Nat. Nanotechnol. 2009, 4, 56-63.

29. Li, Y. Characterization of Engineered Nanomaterials by Spectroscopic Ellipsometry. Available online: http://www.horiba.com/ fileadmin/uploads/Scientific/Documents/TFilm/AN_SE26_Nanomaterials_HD.pdf (accessed on 6 April 2021).

30. Villars, P.; Cenzual, K. Landolt-Börnstein-Group III condensed matter. Landolt Börnstein 2012, 1, 43A11.

31. Landolt, H.; Börnstein, R. Zinc Oxide (ZnO) Refractive Index. Available online: https:/ / materials.springer.com/lb/docs/sm_ lbs_978-3-540-31359-5_305 (accessed on 6 April 2021).

32. Webster, P.J.; Ziebeck, K.R.A. Landolt-Börnstein-Group III condensed matter (Alloys and Compounds of d-Elements with Main Group Elements. Part 2). Landolt Börnstein 1988, 19C. [CrossRef]

33. Kochuveedu, S.T.; Son, T.; Lee, Y.; Lee, M.; Kim, D.; Kim, D.H. Revolutionizing the FRET-based light emission in core-shell nanostructures via comprehensive activity of surface plasmons. Sci. Rep. 2014, 4, 4735. [CrossRef]

34. Herne, T.M.; Ahern, A.M.; Garell, R.L. Surface-enhanced raman spectroscopy of peptides: Preferential n-terminal adsorption on colloidal silver. J. Am. Chem. Soc. 1991, 113, 846-854. [CrossRef]

35. Campion, A.; Kambhampati, P. Surface-enhanced raman scattering. Chem. Soc. Rev. 1998, 27, 241-250. [CrossRef]

36. Dzhagan, V.M.; Valakh, M.Y.; E Raevskaya, A.; Stroyuk, O.; Kuchmiy, S.Y.; Zahn, D.R. Size effects on raman spectra of small CdSe nanoparticles in polymer films. Nanotechnology 2008, 19, 305707. [CrossRef] [PubMed]

37. Schumm, M. ZnO-Based Semiconductors Studied by Raman Spectroscopy: Semimagnetic Alloying, Doping and Nanostructures; Suedwestdeutscher Verlag Fuer Hochschulschriften: Saarbrücken, Germany, 2010.

38. Lautenschlaeger, S.; Eisermann, S.; Meyer, B.K.; Callison, G.; Wagner, M.R.; Hoffmann, A. Nitrogen incorporation in homoepitaxial ZnO CVD epilayers. Phys. Status Solidi (Rrl) Rapid Res. Lett. 2009, 3, 16-18. [CrossRef]

39. Chen, M.C.; Lord, R.C. Laser-excited Raman spectroscopy of biomolecules. VIII. Conformational study of bovine serum albumin. J. Am. Chem. Soc. 1976, 98, 990-992. [CrossRef] [PubMed]

40. Chi, Z.; Chen, X.G.; Holtz, J.S.; Asher, S.A. UV resonance raman-selective amide vibrational enhancement: Quantitative methodology for determining protein secondary structure. Biochemistry 1998, 37, 2854-2864. [CrossRef] [PubMed]

41. Dong, Z.-G.; Liu, H.; Cao, J.-X.; Li, T.; Wang, S.-M.; Zhu, S.-N.; Zhang, X. Enhanced sensing performance by the plasmonic analog of electromagnetically induced transparency in active metamaterials. Appl. Phys. Lett. 2010, 97, 114101. [CrossRef]

42. Ahmadivand, A.; Gerislioglu, B.; Tomitaka, A.; Manickam, P.; Kaushik, A.; Bhansali, S.; Nair, M.; Pala, N. Extreme sensitive metasensor for targeted biomarkers identification using colloidal nanoparticles-integrated plasmonic unit cells. Biomed. Opt. Express 2018, 9, 373. [CrossRef] [PubMed]

43. Toma, M.; Tawa, K. Plasmonic coloration of silver nanodome arrays for a smartphone-based plasmonic biosensor. Nanoscale Adv. 2019. [CrossRef] 